\title{
ORAL HEALTH-RELATED QUALITY OF LIFE IN CHILDREN AND ADOLESCENTS UNDERGOING ANTINEOPLASIC THERAPY: PILOT STUDY
}

\author{
Carrillo $\mathrm{CM}^{1}$, Gianesella M², Rojz JC ${ }^{1}$ \\ ${ }^{1}$ Division of Dentistry, GRAACC-IOP/UNIFESP, São Paulo, Brazil \\ 2 Post graduation student, Department of Pediatrics, Universidade Federal de São Paulo/UNIFESP, São Paulo, Brazil
}

\section{BACKGROUND}

Children and adolescents on antineoplasic therapy are at increased risk for oral and dental complications due to the adverse effects of these therapies on the oral mucosa, altered oral flora, weakening of the immune system and others systems of protection, and by interference with tissue regeneration. ${ }^{1}$ Oral/dental complications may be due to their treatment and/or disease. ${ }^{1,2}$ The most common oral complications include oral mucositis, oral infections, xerostomia, dysgeusia, orofacial pain and trismus. 2 Caries disease and periodontal disease are also commonly detected and are an indirect consequence of antineoplasic treatment through the association of cariogenic diet, changes in oral microflora and inability to maintain good oral hygiene. ${ }^{3,4}$ Oral complications can lead to discomfort and pain, poor nutrition, delays in administration/ limitation of dosages of and increased hospitalization time which may compromise quality of life. ${ }^{1}$

\section{OBJECTIVES}

To assess the oral health related quality of life of children and adolescents undergoing antineoplasic therapy at a pediatric oncology center.

\section{MATERIAL AND METHODS}

Ten patients undergoing treatment for cancer aged 8 to 14 years old were assessed from May 2015 to July 2015. Information collected was: demographic data, medical history, oncologic diagnoses and chief oral complaint. Measures of caries experience (dmft/DMFT) and periodontal disease (Community Periodontal Index) were assessed and calculated. Oral healthrelated quality of life was assessed by the Portuguese validated version of the Child Perceptions Questionnaire (CPQ) in two groups of children and their parents: 8 to 10 years old and 11 to 14 years old. The CPQ presents four subdomains: oral symptoms, functional limitations, emotional and social wellbeing. The questionnaires also contains global ratings of the child's oral health and the extent to which the oral/orofacial condition affected his/her overall wellbeing. The Parental CPQ (P-CPQ) is a measure of parental/ caregiver perceptions of the oral health-related quality of life of children which contains the same four subdomains and overall wellbeing questions of CPQ. Mean number of items scored "Often" or "Every day/ Almost every day" and per cent of subjects with one or more items scored "Often" or "Every day/Almost every day" were calculated. Non parametric test were used to analyze the correlation between the child's perception of his/her oral health and parents' perception of children's oral health and correlation between CPQ scores with CPI and dmft/DMFT.

\section{RESULTS}

Ten patients participated in the study. Mean age of patients was 11.1 years old and $60 \%$ were female. Most of the patients were undergoing chemotherapy $(n=6)$, three were undergoing chemotherapy associated with head and neck radiotherapy and one undergoing head and neck radiotherapy. Xerostomia, dry lips, dysgeusia and orofacial pain were the most common oral complaints among patients. The mean dmft was 4, DMFT 0.9 for mixed and/or permanent dentition and CPI was 2.7. Fifty percent of the subjects aged 8-10 reported experiencing oral symptoms "Often" or "Everyday/Almost everyday" and $60 \%$ of subjects aged 11-14 reported experiencing functional limitations. There were a significant diference between child's perception and parents perception related to subdomain functional limitations on $\mathrm{CPQ}_{8-10}$ group.

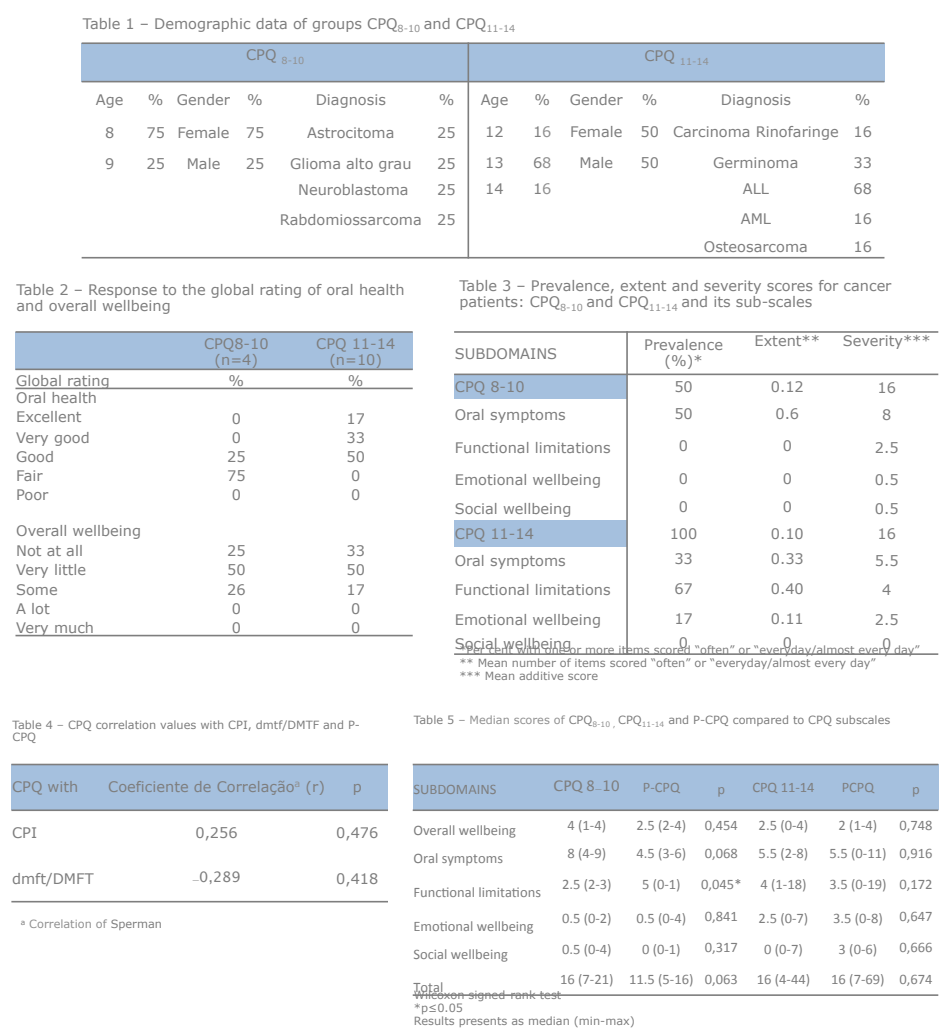

\section{CONCLUSIONS}

Oral complications due to antineoplasic therapy and/or disease may have a negative influence on quality of life of children and adolescents with cancer. Parents view of oral symptoms were significant diferent of their children in group $\mathrm{CPQ}_{8-10}$.

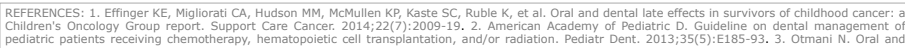
pediatric patients receiving chemotherapy, hematopoietic cell transplantation, and/or radiation. Pediatr Dent. 2013; $35(5)$ ) E185-93.

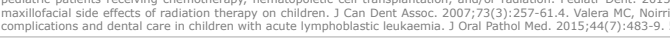

camilamerida@graacc.org.br 